\title{
Investigating the bona fide differentiation capacity of human pluripotent stem cells
}

\author{
Jian-Chien Dominic Heng ${ }^{1,2}$, Kyle M Loh ${ }^{1}$, Huck-Hui Ng${ }^{1,2}$ \\ ${ }^{1}$ Gene Regulation Laboratory, Genome Institute of Singapore, 60 Biopolis Street, Singapore 138672; ${ }^{2}$ Graduate School for Inte- \\ grative Sciences \& Engineering, National University of Singapore, 28 Medical Drive, Singapore 117456 \\ Cell Research (2012) 22:6-8. doi:10.1038/cr.2011.142; published online 30 August 2011
}

Human pluripotent stem cells (hPSCs) have been perennially paraded as a source of cells for cell replacement therapies because they can (theoretically) give rise to any single cell type within the human body [1]. Hence, they can create in vitro a vast number of any human cell type to replace the diseased cell population that a patient might require - this is a salient goal that regenerative medicine aspires to deliver on [2]. However, despite the ever-expanding menagerie of therapeutically relevant differentiated lineages being created from hPSCs, usage of these stem cell-derived progeny for regenerative medicine still remains an uncertainty.

The question here is whether hPSC lines are faithful to their parental pluripotent antecedents in the embryo from which they were derived. During the enterprise of embryonic development, native pluripotent cells housed within the blastocyst give rise to the entire repertoire of differentiated cell types within the human body that successfully subserve important physiological responsibilities. When put to the test, will hPSCs properly differentiate in vitro to produce physiologically functional differenti-

Correspondence: Huck-Hui Ng

Tel: +65 68088145; Fax: +65 680889004

E-mail: nghh@gis.a-star.edu.sg ated cell types that are equatable to the bona fide differentiated lineages that we actually find in the human body? Or will they produce differentiated progeny that partially resemble the cells that we see in vivo? Concerns have surfaced for example, hPSC-derived pancreatic $\beta$-cells sometimes fail to secrete insulin in response to glucose, unlike authentic pancreatic $\beta$-cells found in vivo [3] and hPSC-generated hematopoietic stem cells rarely contribute to the hematopoietic systems of recipient animals [4] - which potentially disqualify usage of these cells to treat diabetes or hematological deficiencies.

In a recent paper published in Cell Research, Patterson et al. investigate the fidelity of differentiated cell types created from hPSCs - were they made in the likeness of actual bodily cells found in the human embryo? To this end, the authors differentiated hPSCs into cell types representative of the three fetal germ layers from which all of the embryo proper arises - neural progenitors (definitive ectoderm), fibroblasts (mesoderm), and hepatocytes (definitive endoderm) - and then they used microarrays to interrogate whether hPSCderived cell types were transcriptionally similar to cells from the same lineage taken from actual human fetuses (Figure 1) [5]. While pervasive transcriptional congruities were found between fetusderived and $\mathrm{hESC} / \mathrm{hiPSC}$-derived cell types, some striking differences were reported - around $\sim 10 \%$ of assayed genes were differentially expressed between embryo-derived and hPSCderived cells (Figure 1). Moreover, no matter if hESCs or hiPSCs were specified into neural progenitors, fibroblasts, or hepatocytes, their resultant differentiated offspring continued to express pluripotency-associated genes LIN28A, LIN28B, and DPPA4, amongst others (Figure 1). Rarely if ever did genuine fetus-derived differentiated cells express any of these three pluripotency markers - hPSC-derived cells generally expressed these genes thousands of times higher than their embryonic counterparts. It is possible that some malingering undifferentiated hPSCs persisted throughout the differentiation protocols, consequently contributing to the elevated expression of these pluripotency genes in hPSC-derived lineages. Whatever the reason, enduring expression of pluripotency genes in stem cell-derived progeny is translationally troublesome; for example, one notes that expression of LIN28 in differentiated cells is tumorigenic [6]. Might hPSC-derived differentiated cells be prone to oncogenic subversion? If so, this could indeed pose a serious impediment to the exploitation of differentiated derivatives of hPSCs for cell-based therapies in human patients.

In light of their findings that hPSC- 


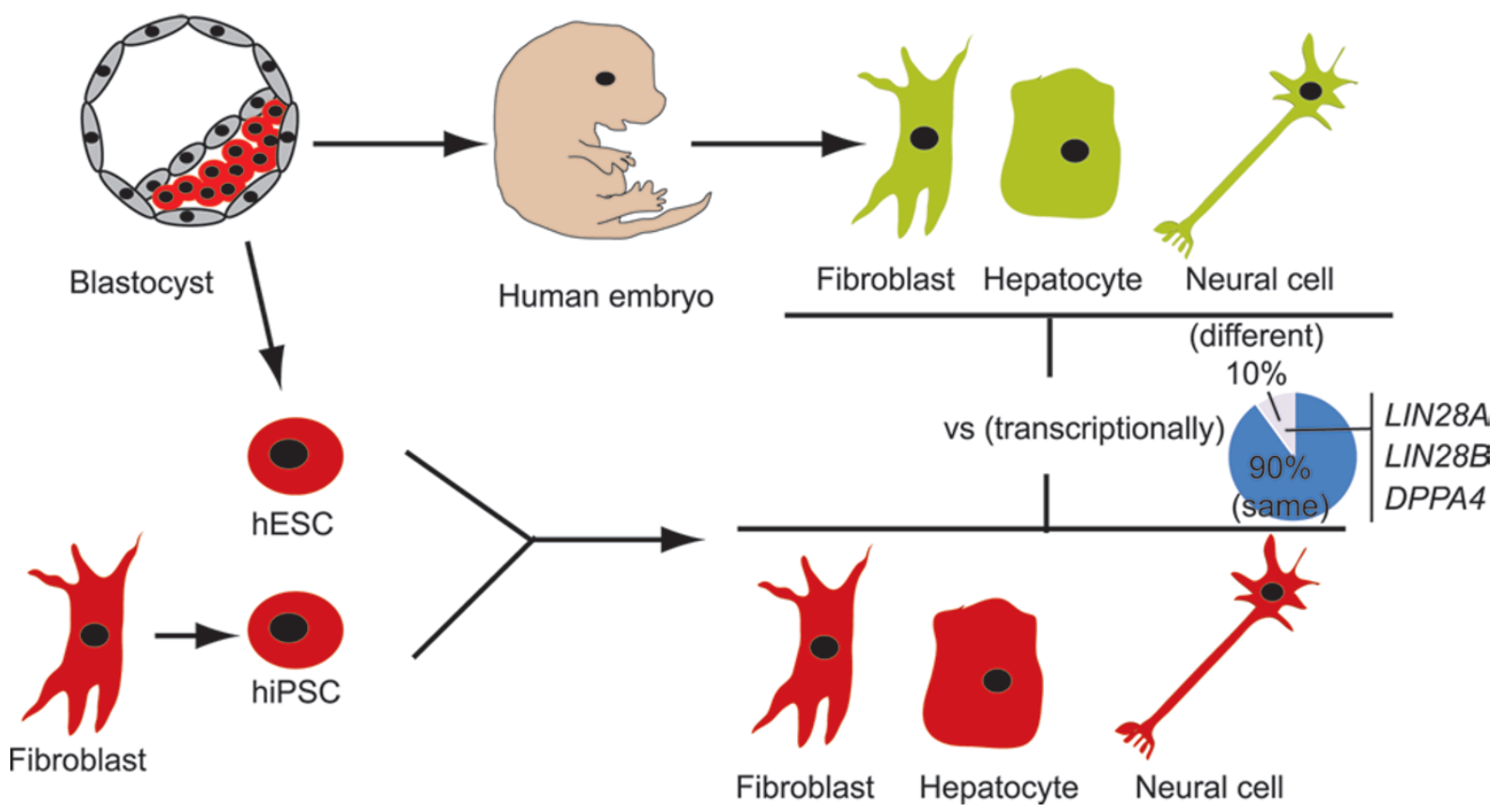

Figure 1 To assess the clinical fitness of differentiated cell types generated from human pluripotent stem cells (hPSCs; both embryonic stem cells and induced pluripotent stem cells), Patterson et al. systematically differentiated hPSCs into neural progenitors, fibroblasts and hepatocytes, and then transcriptionally compared these cells to cells of the same lineage taken from human fetuses (16 weeks old). Microarray analyses found that hPSC-derived cells are generally transcriptionally similar to their embryonic counterparts, although they can differ in the expression of up to $\sim 10 \%$ of the assayed genes. Amongst these differentially expressed genes, it was found that differentiated cells produced from hPSCs generally continue to persistently express pluripotency genes including DPPA4, LIN28A, and LIN28B, motivating concerns about the translational utility of hPSC-derived differentiated cell populations.

derived cells were similar but not identical to bona fide embryo-derived cells, the authors posited that these discrepancies might be an issue of "developmental maturation" - the immediate differentiated progeny of hESCs and hiPSCs might resemble very early differentiated cell types found in the nascent fetus, not more matured differentiated cell types found at later developmental stages. Indeed, this appeared to be partially the case - while hPSC-derived neural progenitors differentially expressed $\sim 10 \%$ of assayed genes as compared to neural progenitors taken from week 16 human fetal spinal cord, the number of differentially expressed genes was halved when they were compared against spinal cord progenitors sourced from weeks 6.5-8 of fetal development (although a subset of genes remained to be differentially expressed regardless of what week of fetal development was chosen). Based on these findings, Patterson et al. advance that hPSC-derived lineages might resemble the respective cell types taken from human fetuses prior to developmental week 6 .

The main import to be abstracted from the findings of Patterson et al. is that hPSC-derived differentiated cell types transcriptionally resemble but are not identical to their embryonic counterparts; and also those hPSC-derived lineages continue to aberrantly express select pluripotency genes. The reasons for these transcriptional incongruities are unclear - for example, we have now become cognizant of the fact that hPSCs in vitro differentially express a large number of genes $(>1700)$ as compared to native pluripotent cells found in human blastocysts [7]. These precocious differences between hPSCs and authentic embryonic pluripotent cells might have contributed to the differences in gene expression seen in their differentiated endpoints - if there are already transcriptional issues with the hPSCs that we are starting out with altogether in the beginning of these in vitro differentiation regimens. Or perhaps hPSCs are not in themselves defective, but our current differentiation procedures are yet to be fully optimized and do not completely recapitulate developmental cell type specification programs found in the embryo, yielding "incompletely programmed" differentiated cell types that are similar yet different from fetal cells [9].

What do these findings portend for the usage of hPSCs for regenerative medicine? Are transcriptional incongruences between hPSC-derived and fetal cells causes for concern? One utilitarian 
argument to be waged is that it is inconsequential that hPSC-derived progeny are transcriptionally divergent from their in vivo counterparts - as long as they can physiologically perform. This also invokes another challenge. The apparent "developmental naïvety" of hPSC-derived cells also remains an important clinical concern - to frame it in the above example of $\beta$-cells; adult $\beta$-cells secrete insulin in response to glucose, but fetal $\beta$-cells do not [3]. Clearly, should hPSCs be capable of differentiating into cell types similar to those found in the fetus, procedures must be elaborated to "mature" these cells into adult-like cells with the appropriate physiological functionalities that are appropriate to transplant into diseased adult patients [8].

Nevertheless, in any event, this present report by Patterson et al. is timely, beckoning concern in regards to the filial piety of hPSCs and their differentiated offspring. This is the first experimental demonstration that there are clear transcriptional differences between multiple
hPSC-derived cell types and their in vivo counterparts. Perhaps further studies delving into their epigenetic differences might yield interesting insights as well. More importantly, whether these cells are still physiologically functional enough to be therapeutically exploited or the differentiation procedures need be improved are the questions now.

\section{References}

1 Thomson JA, Itskovitz-Eldor J, Shapiro SS, et al. Embryonic stem cell lines derived from human blastocysts. Science 1998; 282:1145-1147.

2 Murry CE, Keller G. Differentiation of embryonic stem cells to clinically relevant populations: lessons from embryonic development. Cell 2008; 132:661680.

3 D'Amour KA, Bang AG, Eliazer S, et al. Production of pancreatic hormoneexpressing endocrine cells from human embryonic stem cells. Nat Biotechnol 2006; 24:1392-1401.

4 Tian X, Woll PS, Morris JK, Linehan JL, Kaufman DS. Hematopoietic en- graftment of human embryonic stem cell-derived cells is regulated by recipient innate immunity. Stem Cells 2006; 24:1370-1380.

5 Patterson M, Chan DN, Ha I, et al. Defining the nature of human pluripotent stem cell progeny. Cell Res 2012; 22:178-193.

6 Viswanathan SR, Powers JT, Einhorn $\mathrm{W}$, et al. Lin 28 promotes transformation and is associated with advanced human malignancies. Nat Genet 2009; 41:843-848.

7 Vassena R, Boué S, González-Roca E, et al. Waves of early transcriptional activation and pluripotency program initiation during human preimplantation development. Development 2011; 138:3699-3709.

8 Kroon E, Martinson LA, Kadoya K, et al. Pancreatic endoderm derived from human embryonic stem cells generates glucose-responsive insulin-secreting cells in vivo. Nat Biotechnol 2008; 26:443-452.

9 Wandzioch E, Zaret KS. Dynamic signaling network for the specification of embryonic pancreas and liver progenitors. Science 2009; 324:1707-1710. 